\title{
Globe
}

Revue internationale d'études québécoises

\section{John Chi-Kit Wong : Lords of the Rinks. The Emergence of the \\ National Hockey League, 1875-1936, Toronto, Toronto University Press, 2005}

\section{Pierre-Luc Beauchamp}

Volume 9, numéro 2, 2006

URI : https://id.erudit.org/iderudit/1000887ar

DOI : https://doi.org/10.7202/1000887ar

Aller au sommaire du numéro

Éditeur(s)

Globe, Revue internationale d'études québécoises

ISSN

1481-5869 (imprimé)

1923-8231 (numérique)

Découvrir la revue

Citer ce compte rendu

Beauchamp, P.-L. (2006). Compte rendu de [John Chi-Kit Wong : Lords of the Rinks. The Emergence of the National Hockey League, 1875-1936, Toronto,

Toronto University Press, 2005]. Globe, 9(2), 198-201.

https://doi.org/10.7202/1000887ar d'utilisation que vous pouvez consulter en ligne.

https://apropos.erudit.org/fr/usagers/politique-dutilisation/ 


\section{REVUE INTERNATIONALE D'ÉTUDES QUÉBÉCOISES}

Mais plus fondamentalement, Screening Québec permet de relier, grâce à cette analyse du a cas québécois ", les pratiques populaires à la production cinématographique : elles se rejoignent dans le cinéma direct et fusionnent dans les films du programme Société Nouvelle (puisque la production et la diffusion du film procèdent toutes deux d'une même sphère publique alternative). C'est d'une lumière nouvelle que Scott MacKenzie éclaire ce cinéma atypique, où la production de sens se prolonge dans la salle et influence en retour les cinéastes, englobant public et producteurs dans un même processus de codéfinition.

Gwen Scheppler Université de Montréal

Université Lyon 2

\section{John Chi-Kit Wong}

Lords of the Rinks. The Emergence of the National

Hockey League, 1875-1936

Toronto, Toronto University Press, 2005.

Dans l'univers encore restreint des publications en histoire du sport, le livre de John Chi-Kit Wong tranche avec la tradition historiographique qui domine la production des vingt dernières années. Délaissant le terrain très étudié de l'anthropologie culturelle, Wong aborde l'histoire de la Ligue nationale de hockey (LNH) sous l'angle de la commercialisation et de la professionnalisation. Il n'est donc pas ici question de l'importance symbolique du sport comme rituel ou comme spectacle de masse. Grâce à une lecture nouvelle des auteurs en histoire du sport et à l'analyse des archives de la LNH, Lords of the Rinks raconte plutôt l'histoire d'un groupe d'hommes dont la passion pour le hockey s'est progressivement muée en une volonté de contrôle du marché et d'investissements fructueux, donnant naissance à un empire sportif nordaméricain au seuil de la Deuxième Guerre mondiale.

Ainsi, pour Wong, la genèse de la LNH repose d'abord sur des luttes de pouvoir et d'influence, qui ont généré les principaux éléments structurants de cette organisation. Il identifie également l'axe majeur de développement de la LNH comme étant le passage d'un spectacle de sportifs 


\section{RECENSIONS}

amateurs à une entreprise capitaliste présentant les performances d'athlètes professionnels. Parmi les autres aspects notables dont Wong analyse l'importance, on retrouve l'instabilité qui caractérise l'organisation du hockey jusqu'à la Première Guerre mondiale, le rôle de certains bâtisseurs et hommes d'affaires, ainsi que l'évolution de la LNH vers une américanisation croissante de son capital et de sa structure administrative.

Les premiers chapitres de ce livre sont consacrés aux balbutiements du hockey, d'abord pratiqué par l'élite universitaire de la société canadienne. L'auteur démontre que l'organisation de ce sport en ligues se fait autour de quatre principaux acteurs : les consommateurs de loisirs, les directeurs des clubs de hockey, les médias et, surtout, les propriétaires des patinoires. Il est à noter que les joueurs eux-mêmes ne sont pas inclus dans cette liste. Ici, Wong fait clairement ressortir l'importance des médias, qui jouent déjà un rôle crucial dans le monde du sport-spectacle de la fin du $x^{e}$ siècle (p. 18); mais ce qu'il nous apprend de plus significatif sur le développement du hockey organisé réside dans ce qu'il nomme "the atbletic war", c'est-à-dire le conflit idéologique portant sur l'amateurisme dans le hockey. En effet, là se trouve le creuset du hockey tel que nous le connaissons. Par son statut grandissant de spectacle commercial, le hockey du début du $\mathrm{xx}^{e}$ siècle se professionnalise peu à peu : des joueurs amateurs côtoient des joueurs rémunérés dont la situation ressemble de plus en plus à celle d'employés contractuels (p. 43). Wong identifie ce clivage comme étant à l'origine de la formation de plusieurs ligues concurrentes, dont la plupart gravitent autour de la ville de Montréal, qui demeure le centre névralgique du hockey à cette époque.

Contrairement à ce que l'on pourrait déduire de prime abord d'une approche centrée sur les structures administratives ou économiques, l'auteur ne néglige pas le sport lui-même, ce qui est certainement l'une des forces de son livre. Ainsi, il montre clairement le rôle de la victoire et du talent sportif dans le processus de professionnalisation. Par exemple, l'importance grandissante de la victoire dans les recettes des propriétaires de patinoires et de clubs amène ces derniers à payer de plus en plus les joueurs et à tenter de contrôler un plus grand bassin de joueurs d'élite potentiels.

À la fin de l' "atbletic war ", les joueurs sont des contractuels travaillant pour des clubs, dont les propriétaires se font concurrence pour 
attirer le public. Cependant, une autre guerre est déjà en cours, avant même celle de 1914-1918. En effet, l'élargissement du marché et la popularité grandissante du hockey au Canada donnent lieu à la création de plusieurs ligues, qui regroupent chacune ses propres clubs et qui se font concurrence pour le contrôle du marché canadien. En quelques chapitres, et malgré une certaine lourdeur dans l'explication des détails administratifs, Wong dresse le portrait de cette lutte économique dans laquelle des hommes d'affaires tentent de créer un monopole stable et centralisé afin de rentabiliser leurs investissements. C'est d'ailleurs ce qui se produit, avec l'émergence de la LNH comme puissance organisatrice du hockey. C'est aussi durant cette période que l'auteur remarque le début de l'identification des Canadiens à ce sport (p. 55).

Wong s'attache ensuite à décrire l'expansion de la LNH et du hockey professionnel vers les États-Unis. Durant les années 1920, la LNH profite de la prospérité pour conquérir les grands marchés des villes industrielles du Nord-Est américain. En même temps, les hommes d'affaires canadiens perdent peu à peu le contrôle de la ligue au profit du grand capital américain. L'ouvrage retrace les luttes d'influence qui parsèment cette expansion, notamment celle qui oppose les dirigeants de la LNH à Edward Livingstone (p. 73). Ce conflit est d'ailleurs, selon Wong, l'un des moteurs de l'expansion de la LNH, appuyant ainsi sa thèse initiale du conflit comme générateur de l'évolution. Il est par ailleurs intéressant de constater que cette expansion s'apparente grandement à celle qui a marqué les années 1990 et 2000 dans la LNH : parmi les aspects communs, on peut identifier notamment l'augmentation des coûts de fonctionnement des équipes (déplacements et salaires) et la volonté d'établir un contrôle des coûts.

Les derniers chapitres du livre concernent la consécration de la LNH comme un monopole solide et stable, malgré quelques tentatives d'établir des ligues rivales et en dépit de la crise économique. Wong explique cette réussite par le capital accumulé par l'organisation et par l'établissement d'un système de ligues mineures, qui, tout en assurant un afflux de joueurs dans les équipes de la $\mathrm{LNH}$, permet à cette dernière de contrôler une plus grande part du marché nord-américain sans avoir à investir de gros capitaux dans chacune des villes où opère ce système (p. 137).

Dans l'ensemble, Lords of the Rinks est un livre important, en ce sens qu'il permet de lier les aspects sportifs du hockey à son développement 
comme entreprise commerciale. C'est aussi un ouvrage soigné et empreint d'impartialité, qui tient compte de facteurs humains tels les conflits de personnalité des hommes d'affaires impliqués, mais qui s'attache aussi à comprendre les grandes stratégies commerciales et l'incidence de la conjoncture sur le développement du hockey. Du reste, on ne saurait reprocher à l'auteur de chercher à saisir le rôle des hommes d'affaires dans le développement du hockey, ces derniers ayant encore aujourd'hui la haute main sur les destinées de ce sport. Par ailleurs, on retiendra que le conflit joue un rôle important pour l'auteur. Le débat autour de l'amateurisme, la concurrence entre les ligues, la lutte pour le marché américain : ces conflits sont pour lui autant de facteurs déterminants dans l'évolution du hockey et de la LNH. Il faudra cependant faire un certain travail sur le plan de l'identification à ce sport et de la culture du hockey, spécialement en ce qui concerne sa diffusion dans les masses, afin de compléter ce tableau amorcé sous l'angle des luttes de pouvoir et de l'économie.

Pierre-Luc Beauchamp Université McGill

\section{Pascal Durand, Pierre Hêbert, Jean-Yves Mollier et François Vallotton [éd.] \\ La censure de l'imprimé. Belgique, France, Québec et Suisse romande, $X I X^{e}$ et $X X^{e}$ siècles \\ Québec, Nota bene, 2006.}

Coïncidence ou manifestation d'une conscience inquiète en ces heures de Patriot Act, de rectitude politique, de convergence et de contestation du droit à la liberté de parole, les études sur la censure investissent la place publique. Aux côtés de l'excellent deuxième volet de Censure et littérature au Québec de Pierre Hébert et Élise Salaün ${ }^{14}$, et

14. Pierre HÉBERT, avec la collaboration d'Élise SALAƯN, Censure et littérature au Québec. Des vieux couvents au plaisir de vivre (1920-1959), Montréal, Fides, 2004. 